\title{
Neuralgia pós-herpética: dois casos clínicos
}

\author{
Postherpetic neuralgia: two clinical cases \\ Neuralgia postherpética: dos casos clínicos
}

Daniel Serrano. Centro de Saúde de Portalegre. Unidade de Saúde Familiar Plátano. Portalegre, Portugal. danielcollantes@hotmail.com (Autor correspondente) Teresa Maya Valente. Centro de Saúde de Portalegre. Unidade de Saúde Familiar Plátano. Portalegre, Portugal. teresa.maya@hotmail.com

\section{Resumo}

A neuralgia pós-herpética (NPH) é a complicação mais frequente do herpes-zóster, caracterizando-se pela presença de dor tipo neuropática de distribuição dermatômica, após manifestação prévia de um quadro agudo de herpes-zóster, geralmente um mês depois do surgimento das vesículas. 0 objetivo do presente relato é apresentar dois casos clínicos de NPH e suas respostas a diferentes tratamentos. 0 primeiro caso apresentou intolerância à amitriptilina e uma boa resposta clínica à gabapentina na dosagem de $900 \mathrm{mg} / \mathrm{dia}$. 0 segundo caso apresentou intolerância à capsaicina, mas apresentou boa resposta à combinação da pregabalina com lidocaína tópica. A gabapentina, a pregabalina e a lidocaína tópica são tratamentos eficazes para o tratamento da neuralgia pós-herpética. Todas essas recomendações vão ao encontro dos tratamentos utilizados pelos médicos de família nos dois casos apresentados neste estudo.

\section{Abstract}

Postherpetic neuralgia (PHN) is the most common complication of Herpes Zoster, characterized by the presence of neuropathic-type pain limited to a dermatomic area after outbreak of Herpes Zoster (commonly known as shingles), usually a month after the appearance of vesicles on the skin. The aim of this report is to present two cases of PHN and their responses to different treatments. The first case presented intolerance to amitriptyline and a good clinical response to gabapentin at a dose of $900 \mathrm{mg} / \mathrm{day}$. The second case showed intolerance to capsaicin but responded well to the combination of pregabalin and topical lidocaine. Gabapentin, pregabalin and lidocaine are effective for the treatment of postherpetic neuralgia. All these recommendations are consistent with those treatments used by the family physicians in the two case studies presented.

\section{Resumen}

La neuralgia postherpética (NPH) es la complicación más común de herpes zoster, que se caracteriza por la presencia de dolor tipo neuropático de distribución dermatómica después de una manifestación previa de cuadro agudo de herpes zoster, generalmente un mes después de la aparición de las vesículas en la piel. El objetivo de este relato es presentar dos casos de NPH y sus respuestas a diferentes tratamientos. El primer caso presento intolerancia a la amitriptilina y una buena respuesta clínica a la gabapentina en la dosis de $900 \mathrm{mg} / \mathrm{día}$. El segundo caso mostró intolerancia a la capsaicina, pero mostró una buena respuesta a la combinación de pregabalina associado con lidocaína tópica. La gabapentina, pregabalina y la lidocaína son eficaces para el tratamiento de la neuralgia postherpética. Todas estas recomendaciones son coherentes con los tratamientos utilizados por el médico de familia en dos casos presentados en este estudio.

\section{Palavras-chave:}

Neuralgia Pós-Herpética Herpes Zoster Condutas Terapêuticas Dor

\section{Keywords:} Neuralgia, Postherpetic Herpes Zoster Therapeutical Approaches Pain

Palabras clave: Neuralgia Postherpética Herpes Zóster Conductas Terapéuticas Dolor 


\section{Introdução}

O vírus Varicella-zoster, em um primeiro contato com o paciente, provoca a varicela e, após resolução do quadro, permanece localizado nos gânglios raquidianos posteriores ou nos gânglios geniculado e trigêmeo. ${ }^{1}$ Pode então surgir uma reativação, avançando pela raiz nervosa até o respectivo dermátomo, onde surgirão as lesôes causadas pelo herpes-zóster, a chamada "zona", como é referido muitas vezes pelos pacientes. ${ }^{2,3}$ As causas da reativação não estão ainda bem esclarecidas, mas esta ocorre nos casos de imunidade celular diminuída, como em indivíduos idosos, ou submetidos a tratamentos quimioterápicos, radioterápicos, com corticosteroides, transplantados ou com infecção por HIV. A infecção por herpes-zóster tem uma incidência anual de 215 casos/100.000 habitantes/ano nos EUA ${ }^{4}$ e de aproximadamente 300 casos/100.000 habitantes/ano na Europa, podendo chegar a 1.000 casos/100.000 habitantes/ano em pacientes acima dos 80 anos. ${ }^{5}$

A complicação mais frequente da infecção por herpes-zóster é a dor neuropática pós-herpética - ou neuralgia pós-herpética $(\mathrm{NPH})$ - que surge em até 70\% dos pacientes. ${ }^{6} \mathrm{~A} \mathrm{NPH}$ é causada pelas alteraçóes provocadas pelo vírus herpes-zóster nas estruturas nervosas afetadas. Há um consenso relativo à definição de herpes-zóster, porém o mesmo não ocorre no caso da dor neuropática pós-herpética (NPH) ou neuralgia pós-herpética. As variaçôes na definição da NPH, segundo diferentes autores, refletem-se também nos valores de sua prevalência, tornando difícil comparar diferentes estudos. Em pacientes com idades superiores a 60-70 anos, a prevalência é maior, sendo pouco frequente abaixo desta faixa etária sem que haja outro fator desencadeante. ${ }^{7,8}$

$\mathrm{Na}$ apresentação clínica da NPH, a dor manifesta-se em um dermátomo, com sensação de queimadura, hiperalgesia, hiperestesia ou alodínia. Com a apresentação destes dois casos clínicos, pretende-se refletir sobre a importância dessa complicação, a mais frequente, do herpes-zóster. Esses casos foram selecionados levando-se em consideração:

- o fato de a NPH se manifestar com maior frequência em pacientes acima dos 60 anos e sem outras complicaçóes associadas ao herpes-zóster;

- a continuidade no seguimento da doença ao longo do tempo pelo médico de família;

- as dificuldades que por vezes ocorrem durante o tratamento, responsáveis pela frequente falta de resposta ou efeitos secundários a diferentes condutas terapêuticas.

\section{Descrição do primeiro caso clínico}

\section{Identificação, contexto familiar, hábitos e antecedentes}

Paciente do sexo feminino, raça caucasiana, 78 anos, viúva, aposentada, natural e residente de Portalegre, com 4 anos de escolaridade. O problema de saúde ativo era alteração do metabolismo dos lipídios, e a paciente estava medicada com rosuvastatina $10 \mathrm{mg}$ (uma vez ao dia). Antecedentes familiares sem relevância.

\section{História da doença atual}

A paciente recorreu à consulta de urgências do Centro de Saúde em várias ocasiōes por dor na região dorsal. Ao exame, apresentava vesículas disseminadas características de lesão por herpes-zóster, fechando o diagnóstico. Foram prescritos valaciclovir $1000 \mathrm{mg}, 1$ comprimido a cada 8 horas, e metamizol $575 \mathrm{mg}, 1$ comprimido a cada 8 horas. Posteriormente, foi acrescentado tramadol $50 \mathrm{mg}$ a cada 12 horas devido à dor intensa e persistente. Em outra consulta, foi prescrita lidocaína a $5 \%$ em forma de emplastro medicamentoso e amitriptilina 10mg (Quadro 1). 
Quadro 1. Condutas terapêuticas utilizadas nos casos clínicos.

\begin{tabular}{|cll|}
\hline Consultas & $1^{0}$ Caso clínico & $2^{\circ}$ Caso clínico \\
\hline $1^{\text {a }}$ & Valaciclovir + Metamizol & Valaciclovir + Metamizol \\
$2^{\text {a }}$ & Tramadol & Cianocobalamina \\
$3^{\text {a }}$ & Lidocaína + Amitriptilina & Pregabalina $300 \mathrm{mg}$ \\
$4^{\mathrm{a}}$ & Gabapentina $900 \mathrm{mg}+$ UMP. Retirada de Lidocaína + Amitriptilina & Cianocobalamina + Capsaícina+ Pregabalina 300mg \\
$5^{\mathrm{a}}$ & Gabapentina $1200 \mathrm{mg}+$ UMP & Retirada da Capsaícina posteriormente \\
$6^{\mathrm{a}}$ & Gabapentina $600 \mathrm{mg}$ & Lidocaína + Pregabalina 300mg \\
$7^{\mathrm{a}}$ & Gabapentina $200 \mathrm{mg}$ & \\
\hline
\end{tabular}

A paciente recorreu pela primeira vez ao seu médico de família (Figura 1) referindo náo ter tolerado a amitriptilina, por sedação excessiva, e não apresentar melhorias da dor com a lidocaína. Foi diagnosticada pelo seu médico de família como tendo neuralgia pós-herpética (NPH) e foi entáo prescrita gabapentina $300 \mathrm{mg}$ em dose crescente (começando com 1 comprimido por dia nos três primeiros dias e aumentando até chegar a 1 comprimido a cada 8 horas) e nutrientes com uridina monofosfato (UMP), vitamina $\mathrm{B}_{12}$ (cianocobalamina) e ácido fólico, com uma posologia de 1 comprimido a cada 24 horas. Foi marcada nova consulta em 20 dias para reavaliar a situação clínica. Posteriormente, a paciente começou a diminuir a posologia da gabapentina por iniciativa própria (ao ter diminuído a dor), tendo recebido alta em nove meses (Figura 2).

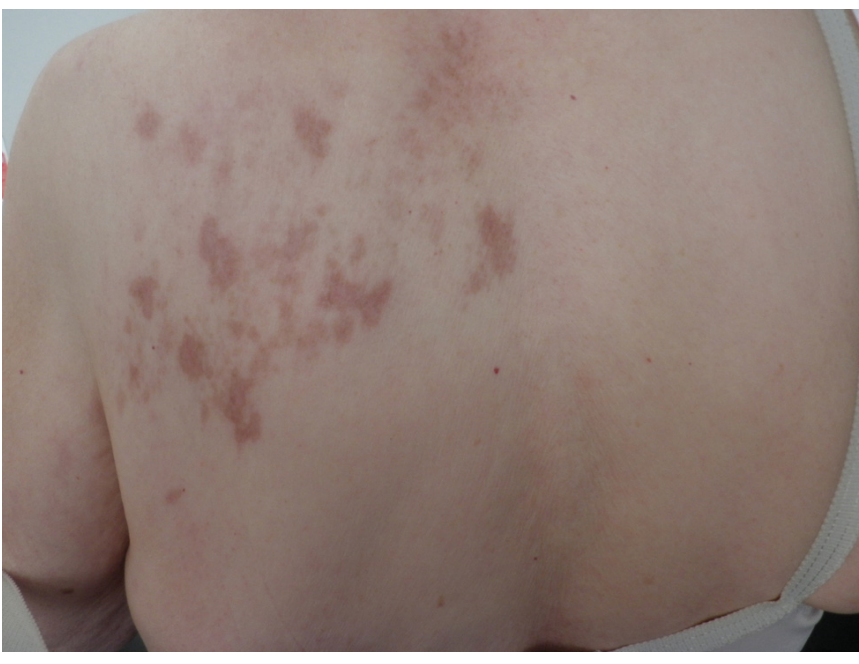

Figura 1. Placas cutâneas acastanhadas na região afetada com dor à palpação.

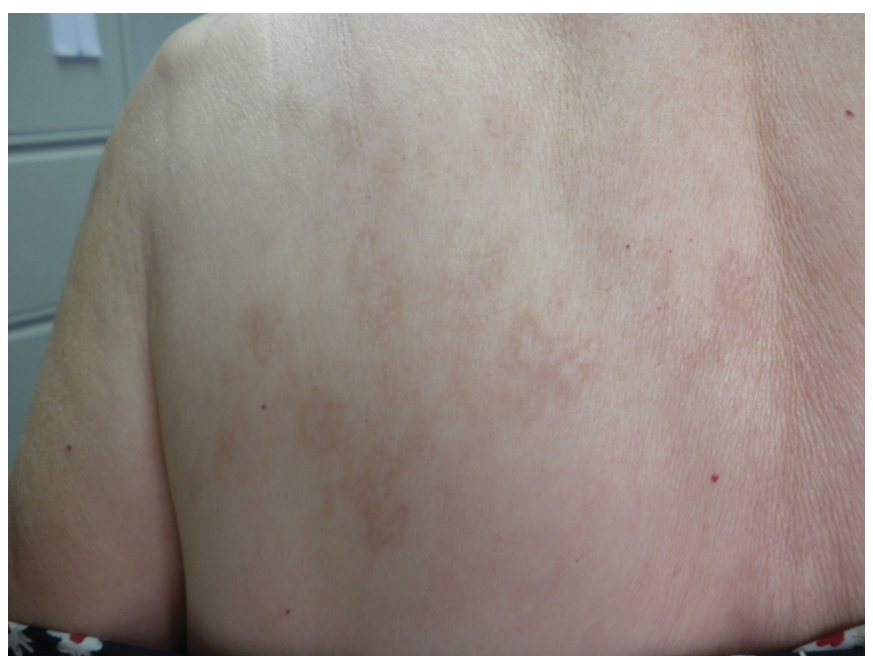

Figura 2. Placas quase imperceptíveis passados nove meses, sem dor.

\section{Descriçao do segundo caso clínico}

\section{Identificação, contexto familiar, hábitos e antecedentes}

Paciente do sexo masculino, raça caucasiana, 81 anos, casado, aposentado, natural de Portalegre, com 4 anos de escolaridade. Os problemas de saúde ativos eram: status pós acidente vascular-cerebral (AVC), hipertensão arterial, doença de Parkinson, síndrome vertiginosa, alterações de memória, déficit de acuidade visual e síndrome depressiva leve. Estava medicado com: idebenona 45mg, 1 comprimido 1 vez ao dia; ácido acetilsalicílico 150mg, 1 comprimido 1 vez ao dia; amitriptilina $25 \mathrm{mg}$, 1 comprimido 1 vez ao dia; beta-histina $24 \mathrm{mg}, 1$ comprimido 2 vezes ao dia; enalapril $20 \mathrm{mg}+$ lercanidipina $10 \mathrm{mg}$, 1 comprimido $1 \mathrm{vez}$ ao dia; levadopa $100 \mathrm{mg}$ + cardidopa $25 \mathrm{mg}, 1$ comprimido $1 \mathrm{vez}$ ao dia; mexazolam $1 \mathrm{mg}$, 1 comprimido 1 vez ao dia; e pantoprazol $20 \mathrm{mg}, 1$ comprimido 1 vez ao dia. Antecedentes familiares sem relevância. 


\section{História da doença atual}

O paciente foi à consulta do seu médico de família queixando-se de dor do tipo queimação, com ardor e picadas de predomínio noturno na região lombar direita e coxa direita, após apresentar herpes-zóster. Relata ter realizado tratamento com vitamina $B_{12}$ (cianocobalamina) $1 \mathrm{mg} / \mathrm{ml}$, sem melhorias. Foi prescrita pelo seu médico de família pregabalina $150 \mathrm{mg}$ duas vezes ao dia. O paciente manteve as queixas de dor neuropática na região referida e retornou ao médico de família, que prescreveu então vitamina $B_{12}$ (cianocobalamina) $1 \mathrm{mg} / \mathrm{ml}$ e capsaína $0,25 \mathrm{mg} / \mathrm{g}$ em creme. Recorreu ao médico de família por apresentar intolerância à capsaína (como "diabo no corpo" sic) e foi prescrito creme de lidocaína $40 \mathrm{mg} / \mathrm{g}$ para aplicar na região, mantendo-se pregabalina $150 \mathrm{mg}$ duas vezes ao dia, sendo que o paciente referiu posteriormente um grande alívio e que a medicação o ajudava a descansar à noite (Quadro 1).

\section{Discussão}

Nos casos relatados, uma paciente de 78 anos e um paciente de 81 anos apresentaram neuralgia pós-herpética (NPH) após episódio de herpes-zóster, fato que coincide com a literatura científica que diz que as complicaçóes do herpes-zóster aumentam com a idade, sendo a neuralgia pós-herpética a complicação mais frequente do herpes-zóster. ${ }^{7,8}$

É importante reconhecer rapidamente o caso de herpes-zóster e administrar um tratamento precoce com agentes antivirais nas primeiras 48-72 horas. ${ }^{9}$ A administração precoce de amitriptilina ou gabapentina também está associada a uma diminuição da frequência de aparecimento subsequente de NPH. O primeiro caso clínico apresentado confirmou a utilidade da gabapentina como um tratamento eficaz e seguro para a dor na neuropatia pós-herpética. No segundo caso clínico, desde a terceira consulta foi utilizada pregabalina na dose de $300 \mathrm{mg} /$ dia, sendo a mesma bem tolerada. Além disso, a combinação da pregabalina com lidocaína tópica em creme $40 \mathrm{mg} / \mathrm{g}$ parece ter resultado em um melhor alívio da dor.

A norma da Direção Geral de Saúde de Portugal (Norma no 43/2011 de 23/12/2011) ${ }^{10}$ refere que na dor neuropática pós-herpética os antidepressivos tricíclicos $(\mathrm{ADT})$ e os gabapentinóides (gabapentina e pregabalina) são preconizados como a primeira opção terapêutica (Nível de Evidência A, Grau de Recomendação I). ${ }^{11,12}$ Em pacientes idosos, a lidocaína tópica pode ser considerada como primeira linha, dada a sua excelente tolerabilidade, em especial se existir preocupaçấo com efeitos adversos no sistema nervoso central associados à terapêutica oral (Nível de Evidência B, Grau de Recomendação I). ${ }^{13}$ Isso coincide com o que algumas diretrizes de prática clínica referem: a) o sumário do Uptodate, ${ }^{14}$ após revisar as evidências e com base na experiência dos autores, recomenda iniciar o tratamento com antidepressivos tricíclicos; b) o Clinical Evidence ${ }^{15}$ classifica as intervençóes sobre neuralgia pós-herpética como efetivas e como drogas de primeira escolha os antidepressivos tricíclicos e a gabapentina.

Portanto, os ADT são considerados fármacos de primeira eleição sempre que não existirem contraindicaçóes específicas do uso. Esses fármacos possuem um efeito central sobre a transmissão da dor, bloqueando a recaptação de noradrenalina e serotonina. $\mathrm{O}$ mais utilizado é a amitriptilina, sendo iniciada pela dose mais baixa (10 a $25 \mathrm{mg}$ em dose única à noite) aumentada semanalmente até uma única dose noturna de $75-100 \mathrm{mg} \cdot{ }^{16}$ Os efeitos secundários mais frequentes são sedação excessiva, hipotensão ortostática e efeitos anticolinérgicos (xerostomia, obstipação intestinal, sudorese excessiva, retenção urinária, visão turva), especialmente em indivíduos com idade avançada. No primeiro caso, o uso da amitriptilina apresentou uma excessiva sedação, o que motivou o abandono da medicação pela paciente. Nos idosos, é preferível utilizar a nortriptilina em uma dose inicial de $25 \mathrm{mg}$ administrada a cada 8 horas, que poderá ser aumentada até um máximo de $150 \mathrm{mg} / \mathrm{dia} .{ }^{17} \mathrm{Os}$ efeitos secundários, nomeadamente o efeito sedativo, sáo menos marcados do que os observados com a amitriptilina. $\mathrm{O}$ tratamento deve ser mantido durante um mínimo de 6 a 8 semanas, uma vez que os efeitos benéficos sáo ocasionalmente tardios. Também a imipramina, por causar menos efeitos anticolinérgicos, pode constituir alternativa à amitriptilina. Aconselha-se precauçáo no uso dos ADT em pacientes idosos, especialmente se apresentam patologia cardíaca, pelos seus efeitos secundários potencialmente graves (cardiotoxicidade: hipotensão postural, arritmias, síncopes). ${ }^{18}$

A gabapentina também pode ser considerada como fármaco de primeira escolha. É o anti-convulsionante mais extensamente estudado, proporcionando uma diminuição na intensidade da dor e na necessidade de administração de analgésicos. A dose mínima eficaz é de $900 \mathrm{mg} / \mathrm{dia}$. A titulação deve ser iniciada com $300 \mathrm{mg}$ preferencialmente à noite, com aumentos de $300 \mathrm{mg}$ a cada 3-5 dias, até se atingir uma dose capaz de proporcionar um alívio da dor, que habitualmente é de 900mg/dia, ou até o surgimento de efeitos secundários relevantes. Os efeitos secundários mais frequentes que a gabapentina pode produzir são: náuseas sonolência, tremor, tonturas, ataxia e aumento ponderal, sendo que a dose máxima não deve exceder $3600 \mathrm{mg} / \mathrm{dia} .{ }^{19}$ 
A pregabalina tem uma estrutura similar à gabapentina, mas apresenta um menor número de efeitos secundários do que o observado para a gabapentina, embora com um preço muito mais elevado. Ensaios clínicos randomizados ${ }^{20} \operatorname{com}$ pacientes com NPH demonstraram uma melhoria no sono e uma diminuição na dor com doses de 150 a $600 \mathrm{mg} / \mathrm{dia}$. O tratamento deve ser iniciado com 25 a $75 \mathrm{mg} /$ duas vezes ao dia, ou começar pela administração à noite, com incrementos a cada 3 a 5 dias, até se atingir $150 \mathrm{mg} / \mathrm{dia}$. A dose máxima, se necessária, não deverá exceder os $600 \mathrm{mg} / \mathrm{dia}$.

Uma alternativa que há vários anos tem se revelado útil no alívio da dor neuropática periférica é a uridina monofosfato (UMP), juntamente com a vitamina $\mathrm{B}_{12}$ e o ácido fólico. Essa combinação favorece a regeneração nervosa ao promover a formação de lecitinas, cefalinas e esfingolipídios.

A lidocaína em emplastro constitui uma terapêutica de $2^{\text {a }}$ linha nesta patologia (Nível de evidência B). Apresenta as vantagens de não requerer titulação e ser segura, com baixa absorção sistêmica e ligeiros efeitos locais, devendo ser utilizada por um período de 12 horas a cada 24 horas.

A capsaicina constitui uma terapêutica de 2a linha (Nível de evidência B) para o tratamento da NPH, mas a sua indicação e a complexidade do seu procedimento justificam a sua realizaçáo no âmbito de Unidades de Saúde específicas para tratamento da dor, visto que frequentemente apresentam efeitos adversos locais, tais como dor, eritema, edema, ou prurido. Todas essas recomendaçóes vão ao encontro dos tratamentos utilizados nos dois casos apresentados neste estudo. O Quadro 2 apresenta as recomendaçôes terapêuticas e seus respectivos níveis de evidência e recomendaçáo.

Quadro 2. Tratamentos utilizados nos casos clínicos e grau de recomendação/ nível de evidência

\begin{tabular}{|lc|}
\hline \multicolumn{1}{|c|}{ Tratamentos } & Grau de recomendação/nível de evidência \\
\hline Antidepressivos tricíclicos & $1^{\mathrm{a}}$ Linha/nível de evidência A \\
Gabapentinóides (gabapentina e pregabalina) & $1^{\mathrm{a}}$ Linha/nível de evidência A \\
Lidocaína & $2^{\mathrm{a}}$ Linha/nível de evidência B \\
Capsaícina & $2^{\mathrm{a}}$ Linha/nível de evidência B \\
\hline
\end{tabular}

Fonte: Direção Geral de Saúde de Portugal (Norma nº 43/2011). ${ }^{10}$

\section{Agradecimentos}

O autor agradece a colaboração da Dra. Manuela Santos por ter aportado um dos casos clínicos.

\section{Referências}

1. Mahalingam R, Wellish M, Wolf M. Latent varicella-zoster virus DNA in human trigeminal and thoracic ganglia. N Eng J Med. 1990;323:627-31. http://dx.doi.org/10.1056/NEJM199009063231002.

2. Straus SE, Ostrove JM, Inschuspé G, Felser JM, Freifeld A, Croen KD, et al. Varicella-zoster virus infections. Biology, natural history, treatment, and prevention. Ann Intern Med. 1988;108:221-37. http://dx.doi.org/10.7326/0003-4819-108-2-221.

3. Gilden DH, Kleinschmidt-DeMastersmBK, La Guardia J, Mahaligam R, Cohrs J. Neurologic complications of the reactivation of varicella-zoster virus. N Eng J Med. 2004;342:635-45. http://dx.doi.org/10.1056/NEJM200003023420906.

4. Donahue JG, Choo PW, Manson JE, Platt R. The incidence of herpes zoster. Arch Intern Med. 1995;155(15):1605-9. http://dx.doi.org/10.1001/archinte.1995.00430150071008.

5. Volpi A, Gross G, Hercogova J, Johnson RW. Current management of herpes zoster: the European view. Am J Clin Dermatol. 2005;6(5):317-25. http://dx.doi.org/10.2165/00128071-200506050-00005.

6. Gnann JW Jr; Whitley RJ. Herpes zoster. N Engl J Med. 2002;347(5):340-346. http://dx.doi.org/10.1056/NEJMcp013211.

7. Helgason S, Sigurdsson JA, Gudmundsson S. The clinical course of herpes zoster: a prospective study in primary care. Eur J Gen Pract. 1996;2(1):12-16. http://dx.doi.org/10.3109/13814789609161651.

8. Helgson S, Petursson G, Gudmundsson S, Sigurdsson JA. Prevalence of postherpetic neuralgia after a first episode of herpes zoster: prospective study with long term follow up. BMJ. 2000;321(7264):794-6. http://dx.doi.org/10.1136/bmj.321.7264.794.

9. International Herpes Management Forum. Improving the management of varicela, herpes zoster and zoster-associated pain. Disponível em: http://www.ihmf.org/guidelines/sumary11.asp.

10. Norma da Direção-Geral de Saúde. Terapêutica da Dor Neuropática. Número 43/2001 de 23/12/2011. Disponível em: www.dgs.pt 
11. National Institute for Health na Care Excellence (NICE). CG173: Neuropathic pain: the pharmacological management of neuropathic pain in adults in non-specialist settings. London: NICE; 2013 Nov. Disponível em http://www.nice.org.uk/guidance/CG173.

12. Attal N, Cruccu G, Haanpää M, Hansson P, Jensen TS, Nurmikko T et al. EFNS Task Force. EFNS guidelines on pharmacological treatment of neuropathic pain. Eur J Neurol. 2006 Nov; 13(11):1153-69. http://dx.doi.org/10.1111/j.1468-1331.2006.01511.x.

13. Chen H, Lamer TJ, Rho RH, Marshall KA, Sitzman BT, Ghazi SM, et al. Contemporany management of neuropathic pain for the primary care physician. Mayo Clin Proc. 2004;79(12):1533-1545. http://dx.doi.org/10.4065/79.12.1533

14. Bajwa ZH, Warfield CA, Crovo DG. Postherpetic neuralgia. Waltham (MA): UpToDate, 2014 [acesso em 2013 May 23]. Disponível em: www.uptodate.com/contents/postherpetic-neuralgia?source=search_result\&search=bajwa+postherpetic+neuralgia\&selectedTitle=6 95.

15. Watson PN. Postherpetic neuralgia. Clin Evid. 2010;10:905. PMC3217735.

16. Browsher D. The effects of pre-emptive treatment of postherpetic neuralgia with amytrptiline. A randomized, double-blind, placebo-controlled trial. J Pain Symptom Manage. 1997;12:327-31. http://dx.doi.org/10.1016/S0885-3924(97)00077-8.

17. Watson CPN, Vernich L, Chipman M, Reed K. Nortryptiline versus amytrptiline in postherpetic neuralgia: a randomised trial. Neurology. 1998;51:1166-71. http://dx.doi.org/10.1212/WNL.51.4.1166.

18. Fisterra. Guia famacoterapéutica. Antidepresivos Tricíclicos. [acesso em 2006 May 23]. Disponivel em: http://www.fisterra.com/medicamentos/.

19. Fisterra. Guia farmacoterapéutica. Gabapentina [acesso em 2006 May 23]. Disponível em: http://www.fisterra.com/medicamentos/.

20. Sabatowski R, Galvez R, Cherry DA, Jacquot F, Vincent E, Maisonobe P, et al. Pregabalin reduces pain and improves sleep and mood disturbances in patients with post-herpetic neuralgia: results of a randomised, placebo-controlled clinical trial. Pain. 2004;109:26-35. http://dx.doi.org/10.1016/j.pain.2004.01.001 